\title{
Experimental research on solids conveying capacity of positive pressure up-extraction fluidized bin pneumatic conveying cement powder
}

\author{
DUAN Guangbin ${ }^{a}$, WANG Wenzhi ${ }^{b}$, LIU Feng ${ }^{c}$, LI Jinkai ${ }^{d}$, LIU Zongminge, ${ }^{\text {* }}$
}

School of Material Science and Engineering, China, University of Jinan, Jinan 250022, China

amse_duangb@ujn.edu.cn, ${ }^{\mathrm{b}} \mathrm{mse} \_w a n g w z @ 163 . c o m,{ }^{\mathrm{c}} \mathrm{mse}$ liuf@163.com, ${ }^{\mathrm{d}} \mathrm{mse}$ _lijk@ujn.edu.cn, est_liuzm@ujn.edu.cn ( ${ }^{*}$ : Corresponding author)

\section{Keywords: Positive pressure up-extraction fluidized bin; Pneumatic conveying; Solid conveying capacity}

Abstract. In view of the high requirement of solid phase quantification for pneumatic conveying in some parts of industrial \& agricultural production, positive pressure up-extraction fluidized bin pneumatic conveying system was established. Experiments were carried out in this pneumatic conveying system by transmitting cement powder. The trend of solid conveying capacity during the conveying process was obtained according to the results of precise weighing sensor test and ultrasonic testing method. Through the comparison of solid flow before and after automatic control, it could be seen that the stable conveying time increased 16 18s, meanwhile the fluctuation of solid conveying capacity was less than $\pm 8 \%$ in condition of automatic control status. The results showed that the automatic control scheme improved the stability of solid flow greatly.

\section{Introduction}

Pneumatic conveying was an effective transportation style, which was widely used in power engineering, food industry, building materials [1], chemical industry, metallurgy and other fields. Especially on some certain occasions, pneumatic conveying is unique. The pneumatic conveying of pre-theoretical research and engineering application most focused on uniform gas-solid suspension flow. The main research methods involved experiment test, theoretical research and numerical simulation. The advantages of uniform suspension flow were stable flow, easy to control etc. But meanwhile, high energy consumption, the valve, pipeline and other parts wear serious existed[2]. Therefore, in the engineering application, to explore more efficient and better adaptability of the materials, solid conveying ability of accurate control of pneumatic conveying mode came to a new subject.

Pump type pneumatic conveying mode used widely because of the advantages of good stability, relatively compact and low cost. However, this type of pump system has solid conveying capacity of large fluctuations in transport process, which limited its application in high solid quantitative demanding conditions[3].

In this paper, up-extraction fluidized bin pneumatic conveying system was designed and built up. Gas solid flow experiments were carried out by conveying cement particles. Based on the experimental data, the trend of system conveying capacity, vs. gas velocity, pneumatic pressure etc flow parameters was performed in detail. Meanwhile, auto control system was built up according to experiment process. By adjustment and control, solid flow ratio could be adjusted. Finally, the solid regulation of before and after the conveying capacity are analyzed.

\section{Experimental SET-UP}

In this paper, the conveying tests were performed in a $0.08 \mathrm{~m}$ I.D, $75 \mathrm{~m}$ length pipe flow conveying system. The transporting system (see Fig.1) was made up of gas supply, feeder, collecting system, data acquisition system, and pipeline. 


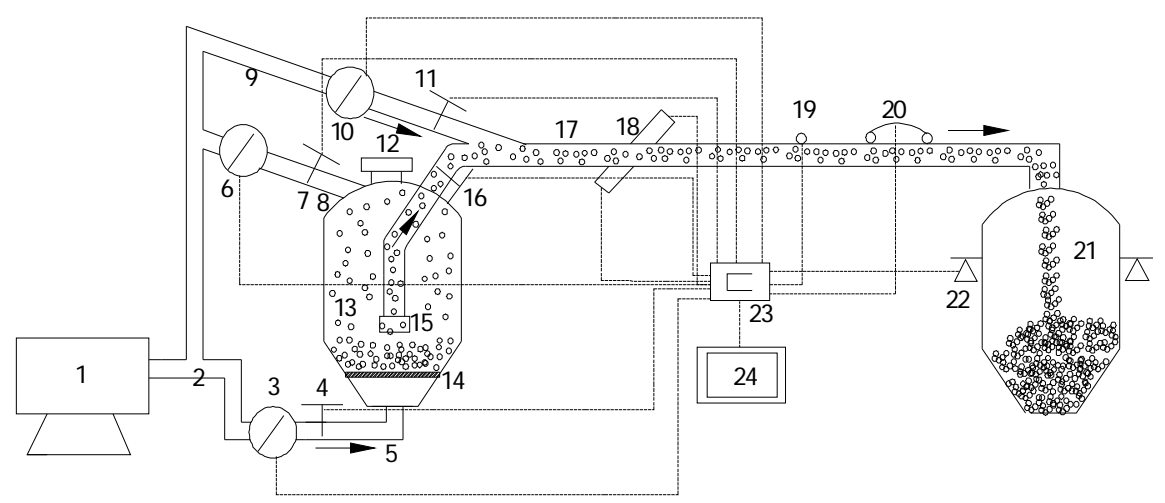

1. gas compressor 2. gas pipeline 3. fluidization gas flowmeter 4. fluidization gas control valve 5. fluidization gas pipeline 6.backpressure gas flowmeter 7. backpressure gas control valve 8.backpressure gas pipeline 9. pusher gas pipeline 10. pusher gas pipeline flowmeter 11. pusher gas pipeline gas control valve 12.feed inlet 13. feeder bin 14.fluidized bed 15.discharge 16. conveying pipeline control valve 17. conveying pipeline 18. ultrasonic test instrument 19. static pressure sensor 20. differential pressure sensor 21. receiving bin 22.weighing sensor 23. data acquisition card 24. computer

Fig.1. Diagram of experimental system

In this paper, cement powder was fed in conveying bin. the feed inlet was closed by butterfly valve. The gas compressor was opened and compressed air entered the feeder bin. Cement was fluidized when the static pressure arrived at a constant value. The gas solid two phase flow entered pipe, and finally reached the receiving bin. In this experiment, the solid particles concentration decreased gradually until no particles in pipe. In process of conveying solids, trend of solid flow ratio by monitoring the change of high accuracy weighing sensors and ultrasonic test was given. Ultrasonic test instrument was installed away $36 \mathrm{~m}$ from the exit. Meanwhile the paper established PID control system with PLC+WINCC. Of course, we can adjust solid mass conveying ratio by setting the opening controller valve of fluidization gas, pusher gas, conveying gas, which can achieve solid flow constantly.

$500 \mathrm{~kg}$ cement was placed in feeder bin, which was about $2 / 3$ of feeder volume. The material was dry and its physical parameters were shown in Table 1.

Table 1 Material properties

\begin{tabular}{ccccc}
\hline Powder & Equivalent spherical diameter $[\mu \mathrm{m}]$ & Particle density $\left[\mathrm{kg} / \mathrm{m}^{3}\right]$ & Bulk density $\left[\mathrm{kg} / \mathrm{m}^{3}\right]$ & Sphericity \\
\hline Cement & 56.81 & 2428 & 1341 & 0.82 \\
\hline
\end{tabular}

\section{Experimental Results}

In this paper, the gas velocity was $12.5 \sim 18 \mathrm{~m} / \mathrm{s}$ and the conveying pressure was set $0.28 \sim 0.35 \mathrm{MPa}$.

\section{Trend of solids conveying capacity when conveying gas was opened.}

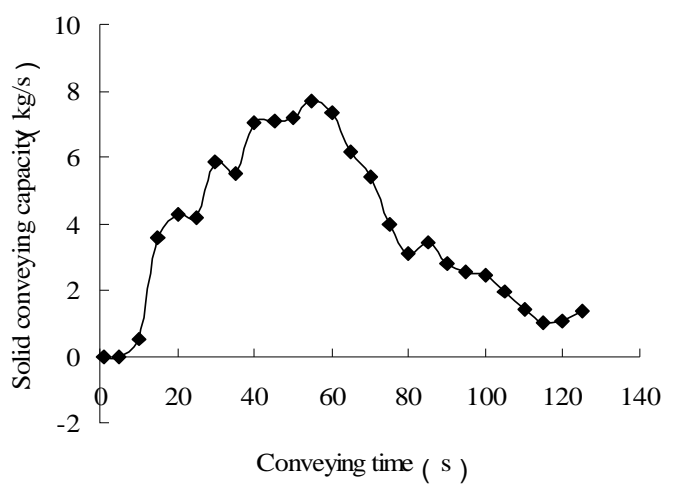

Fig. 2 Trend of solids conveying capacity vs conveying time when conveying gas was opened and weight sensor was adopted

Fig. 2 showed the curve of solid conveying capacity vs. conveying time, as the fluidization gas and conveying pipeline control valves opening when weight sensor was adopted. From this figure, the solid conveying capacity kept 0 as transportation began. After about 12s, the particles arrive at the receiving 
bin, and the weighing sensors displayed. As the conveying continued, the solid conveying capacity increased to the maximum value of $8.0 \mathrm{~kg} / \mathrm{s}$, while the increasing gradient was smaller. It mainly because that gas entered feeder, the solid particles were fluidized fully. Then gas solid two phase flow fixed well. Under the action of the pressure difference, the solid particles enter the conveying pipe. The conveying kept constant value for a moment and then decreased immediately. The stable conveying sustained less, and the rangeability of solid flow ratio was much larger. It was because that, as conveying continued, the solid particles mass concentration decreased, which led to the solid flux reducing down. Simultaneously, the conveying capacity fluctuated greatly because of unstable solid flux. According to the experimental data, the conveying capacity ranged from $3.9 \sim 8.0 \mathrm{~kg} / \mathrm{s}$.

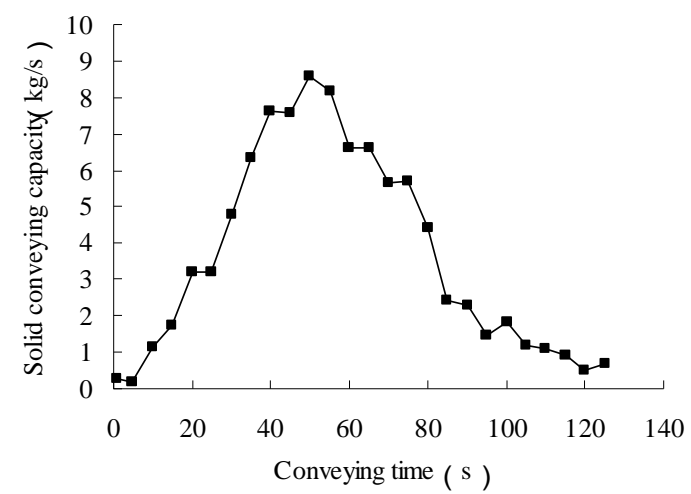

Fig. 3 Trend of solids conveying capacity vs. conveying time when conveying gas was opened and ultrasonic test was adopted

Fig. 3 showed the curve of solid conveying capacity vs. conveying time, as the fluidization gas and conveying pipeline control valves opening when ultrasonic test was adopted. From this figure, at the beginning, the solid conveying capacity kept 0 . After about $6.5 \mathrm{~s}$, the particles arrive at the test points, and the ultrasonic test instrument displayed. As the process went on, the solid mass ratio arrived at 8.9 $\mathrm{kg} / \mathrm{s}$ with high gradient, and then decreased to 0 rapidly. At the same time, when only the fluidizing gas was opened, the transport capacity of solids varies greatly from $3.2 \sim 8.9 \mathrm{~kg} / \mathrm{s}$.

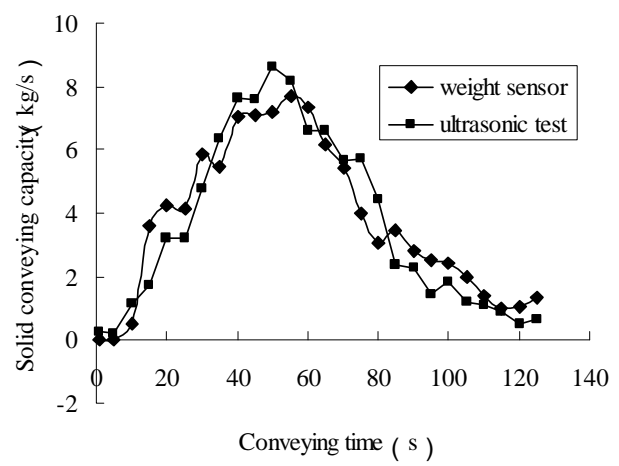

Fig. 4 Trend of solids conveying capacity vs. conveying time when conveying gas was opened and weight sensor, ultrasonic test were adopted

Fig. 4 showed the comparison curve of solid conveying capacity vs. conveying time, as the fluidization gas and conveying pipeline control valves opening when ultrasonic test was adopted. From these curves, two kinds of methods of testing was more similar, especially in the stable section of conveying process, the two curves were closer. Through the relative error analysis, it was found that the maximum relative error of the two test results was found to be less than $10 \%$, which showed the use of weighing sensor and ultrasonic test were used to performance the ability of solid conveying capacity. 


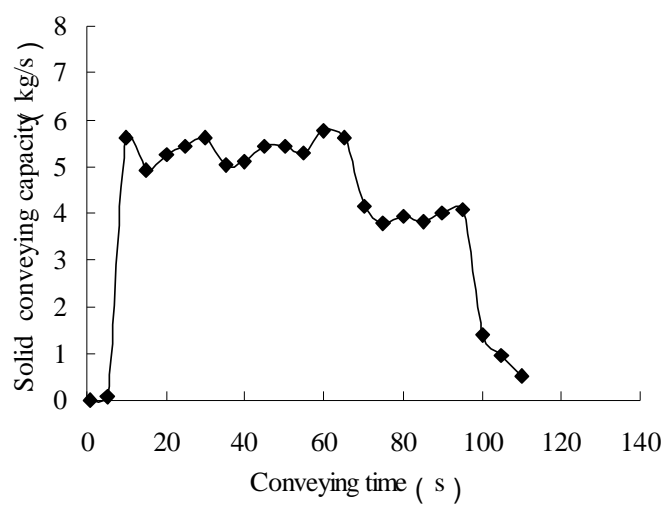

Fig. 5 Trend of solids conveying capacity vs. conveying time when adjustment gas was opened and weight sensor was adopted

Fig. 5 showed the curve of solid conveying capacity vs. conveying time, as the adjustment gas and conveying pipeline control valves opening when weight sensor was adopted. From this figure, the solid conveying capacity kept 0 as transportation began. After about $9 \mathrm{~s}$, the weighing sensors displayed. The solid conveying capacity increased to an averaged value of $5.8 \mathrm{~kg} / \mathrm{s}$, while the increasing gradient was bigger. With conveying proceed, the control valve was opened, the solid conveying capacity kept uniform and maintained at $5.5 \mathrm{~kg} / \mathrm{s}$. From the control system could be seen, when the back compressor valve opened, the pump pressure increased which led to the rate of solid entering the pipeline increasing. When reached a certain moment, the solid particles in the pump decreased. At this point, reducing the opening of the back pressure, transportation capacity decreased rapidly to 0 . In addition, through the comparison of Fig.2, we could see that the stability of solid conveying capacity had been greatly improved. The stable conveying time was maintained at 15 100s, 40s was increased by Fig. 2 . In the meantime, the magnitude of the solid transport capacity had been reduced, between $5.0 \sim 5.4 \mathrm{~kg} / \mathrm{s}$. The maximum relative error from the mean was less than $\pm 8 \%$.

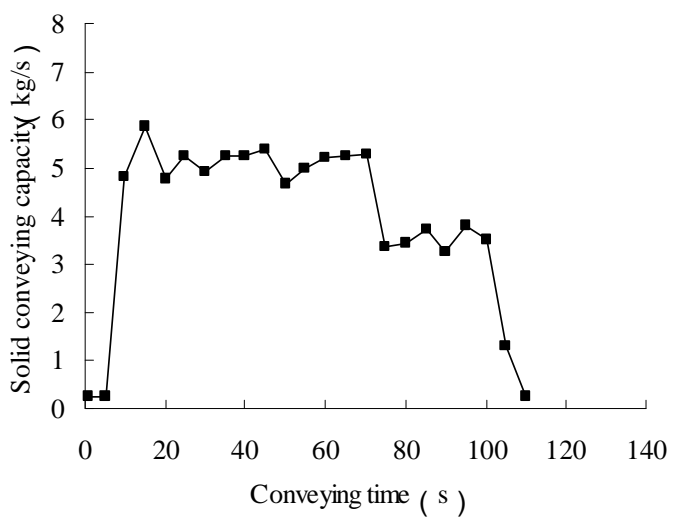

Fig.6 Trend of solids conveying capacity vs. conveying time when adjustment gas was opened and ultrasonic test was adopted

Fig. 6 showed the curve of solid conveying capacity vs. conveying time, as the fluidization gas and conveying pipeline control valves opening when ultrasonic test was adopted. From this figure, the changing trend of solid conveying capacity was consistent with the variation trend of weighing sensor. From this figure, at the beginning, the solid conveying capacity kept 0 . After about $8.5 \mathrm{~s}$, the ultrasonic test instrument displayed. As the process went on, the solid mass ratio arrived at $5.9 \mathrm{~kg} / \mathrm{s}$ with high gradient.

As the delivery continued, the solids conveying capacity remained uniform at $4.9 \mathrm{~kg} / \mathrm{s}$. when the value decreased, the adjustment gas was opened automatically, solid transport capacity was more balanced. While a certain moment was reached, the solid particles in the sump pump become less and eventually decreased to 0 . In addition, through the comparison of Fig.3, we could see that the stability of solid conveying capacity had been greatly improved. The stable conveying time was maintained at 
20 90s, 30s was increased by Fig.3. In the meantime, the magnitude of the solid transport capacity had been reduced, between $5.2 \sim 5.6 \mathrm{~kg} / \mathrm{s}$. The maximum relative error from the mean was less than $\pm 10 \%$.

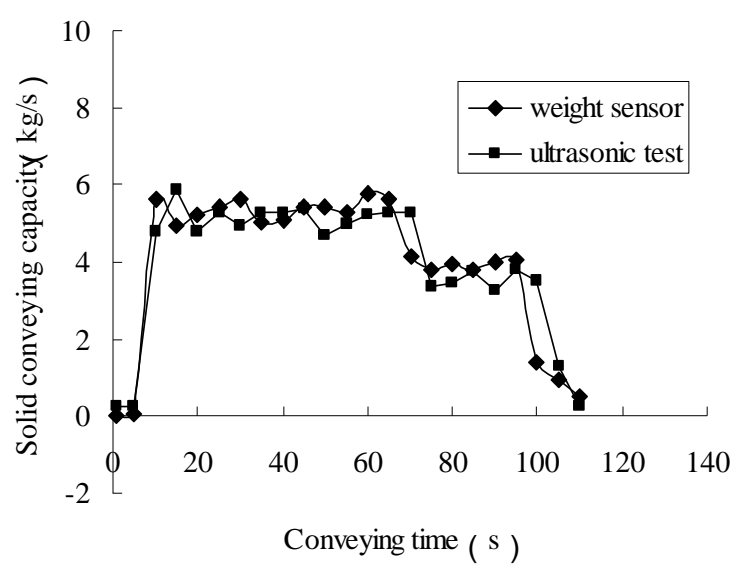

Fig. 7Trend of solids conveying capacity vs. conveying time when adjustment gas was opened and weight sensor \& ultrasonic test were adopted

Fig. 7 showed the comparison curve of solid conveying capacity vs. conveying time, as the adjustment gas and conveying pipeline control valves opening when ultrasonic test was adopted. From these curves, two kinds of methods of testing were more similar. Through the relative error analysis, it was found that the maximum relative error of the two test results was found to be less than $10 \%$ too.

\section{Conclusions}

An industrial scale positive pressure fluidized pump pneumatic conveying system has been established. The system was fully functional, and the real-time monitoring of solid conveying capacity was realized by weighing transducer and ultrasonic testing method. At the same time, the automatic control system of solid conveying capacity had been established. By adjusting the opening of different intake valves, the quantitative adjustment of conveying capacity could be achieved.

The pneumatic conveying experiment using the cement powder as the conveying material and the compressed gas as the conveying power was carried out. The results showed that the capacity of solid conveying increased first and then decreased without auto-control. The stability time was relatively small, and the transport capacity fluctuated greatly. But after using the solid transportation capacity feedback automatic control, the steady conveying time increased by 26-30s, the solid conveying capacity of the whole conveying process was more balanced, and the maximum relative error was less than $\pm 8 \%$. At the same time, the solid conveying capacity of the weighing transducer and ultrasonic test agreed the true results well which proved the two approaches can be used to monitor the solid conveying capacity.

\section{Acknowledgements}

This work was supported by Shandong Provincial Technology Development Project (2013GGX10308), Natural Science Foundation of Shandong Province (ZR2015EL037), Shandong Provincial Technology Development Project (2014GGX105007) and Jinan Technology Development Project (201202071).

\section{References}

[1] X. Zheng, Y. Liu and W. Liu: Ind. Eng. Chem. Res. Vol. 49(22) (2010), p. 11814-11824.

[2] S. Teng, P. Wang and Q. Zhang: Powder Technol. Vol. 208 (2011), p. 684-693.

[3] Y. Cui, H. Gao and J. Sun: Powder Technol. Vol. 226 (2012), p. 34-42. 